\title{
A homoclinic tangle on the edge of shear turbulence
}

\author{
Lennaert van Veen* \\ Faculty of Science, University of Ontario Institute of Technology, \\ 2000 Simcoe St. N., Oshawa, L1H 7K4 Ontario, Canada \\ Genta Kawahara \\ Graduate School of Engineering Science, Osaka University, \\ 1-3 Machikaneyama, Toyonaka, Osaka 560-8531, Japan
}

(Dated: November 8, 2018)

\begin{abstract}
Experiments and simulations lend mounting evidence for the edge state hypothesis on subcritical transition to turbulence, which asserts that simple states of fluid motion mediate between laminar and turbulent shear flow as their stable manifolds separate the two in state space. In this Letter we describe a flow homoclinic to a time-periodic edge state. Its existence explains turbulent bursting through the classical Smale-Birkhoff theorem. During a burst, vortical structures and the associated energy dissipation are highly localized near the wall, in contrast to the familiar regeneration cycle.

PACS numbers: 47.27.Cn, 47.27.ed, 47.52.+j
\end{abstract}

Introduction In recent years, the open problem of subcritical transition to turbulence in shear flows has seen a surge of interest, sparked by a rapidly increasing ability to perform numerical simulations as well as by a string of novel applications of dynamical systems theory. The dynamical systems approach advocates the idea, that transitional shear flow is regulated by special solutions with a relatively simple spatial structure. They may be travelling waves, time-periodic solutions or even solutions chaotic in time. Such states live in between a stable laminar state and stable, or meta-stable, turbulence. In simulations, these special states can be identified by a shooting algorithm in which initial data are iteratively refined to yield a flow which neither laminarizes nor becomes turbulent, but instead lingers on the "laminar-turbulent boundary" [1]. States on this boundary are necessarily unstable and consequently they can only be observed as transient effects in experiments [2]. Their relevance to the transition process lies mainly in their stable and unstable manifolds in state space. These determine how the fluid behaves as it transitions from near-laminar to turbulent states and vice versa.

A particularly interesting situation arises when the laminar-turbulent boundary is formed - at least locally by the stable manifold of a traveling wave or periodic solution, which is then called an "edge state". Edge states have now been computed for flow in channels as well as pipes, with various numerical schemes and spatial discretizations, and there is growing consensus that they are a robust feature of subcritical shear flow [3].

Logically, the next step in this analysis would be to study the stable manifolds of the edge states. Knowledge of the geometry of these manifolds would open the door to the application of control techniques, aiming at a forced laminarization of the flow. Indeed, some results to this effect have been obtained using linearization about an edge state [4]. Little, if anything, is know about the global, nonlinear structure of these separating manifolds, greatly reducing the usefulness and predictive power of the edge state hypothesis. The direct study of a separating manifold is hard if not impossible, owing to its high (formally infinite) dimensionality. In the current Letter, we commence by studying the two-dimensional unstable manifold of an edge state in plane Couette flow, using a novel computational algorithm. We find that it contains an orbit which returns to the edge state along its stable manifold. Through the classical Smale-Birkhof theorem 5], the presence of this homoclinic orbit implies the existence of an intricate tangle of the stable and unstable manifolds and chaotic dynamics which manifests itself as irregular turbulent bursting.

Transitional plane Couette flow We consider plane Couette flow at a Reynolds number of $R e=400$ in the minimal flow unit of dimensions $L \times W \times H$, where $R e$ is based on half the velocity difference between the two walls, $U / 2$, and half the wall separation, $H / 2$. The streamwise and spanwise periods are $(L / H, W / H)=$ $(2.76,1.88)$ [6]. We used resolutions of $16 \times 33 \times 16$ as well as $32 \times 33 \times 32$ grid points in the streamwise $(x)$, wall-normal $(y)$ and spanwise $(z)$ directions, respectively, and checked that the behaviour is qualitatively the same. In this computational domain, no Nagata 7] steady solution exists 8]. Instead, the edge state in this flow is a time-periodic variation of the laminar flow, which shows weak, meandering streamwise streaks [4, 9]. This gentle Unstable Periodic Orbit (UPO) has a single unstable Floquet multiplier and thus its unstable manifold has dimension two and its stable manifold has codimension one in state space. Both the UPO and its unstable manifold are contained in a subspace invariant under the spatial symmetries given by reflection in the mid plane, followed by a streamwise shift over $L / 2$, and reflection in the streamwise and spanwise direction, follow by a spanwise shift over $W / 2$. Consequently, we can impose these 
symmetries on the solutions to reduce the number of degrees of freedom, without placing artificial restrictions on the fluid motion. At the higher resolution, the number of degrees of freedom in the simulations is about 11,000 . Homoclinic orbit computation The unstable manifold of the UPO can be computed using multiple-shooting orbit continuation [10]. Essentially, this algorithm produces a sequence of orbits, contained in the manifold, by arclength continuation of a boundary value problem in time. Because of the extremely sensitive dependence on initial conditions in turbulent Navier-Stokes flow, we need to compose the orbits of multiple segments, each not much longer than the decorrelation time, i.e. the time scale of exponential divergence of turbulent states of fluid motion which are initially close. The results presented below use up to six shooting intervals, the integration time on each interval staying below two times the period $T$ of the UPO and below five times the decorrelation time.

As we compute a sequence of orbits, it may happen that it converges to a homoclinic orbit, which separates the unstable manifold into two components. Such an orbit is shown in Fig. 1(top). In this projection on energy input and dissipation rate, the UPO is the tiny loop labelled $L$. In the background we have plotted the Probability Density Function (PDF) of transient turbulence. In the transition to turbulence the homoclinic orbit overshoots the maximum of the PDF, then passes close to it on the way back to the UPO. Clearly, the shape of the PDF agrees well with that of the homoclinic.

We generically expect the homoclinic orbit to approach the UPO along the least stable subspace of the latter, also called the leading stable subspace. This is visibly the case in Fig 1(bottom), which shows a close-up of the UPO, along with the local unstable manifold in red and the local leading stable subspace in blue, now with a third axis showing the mean square streamwise vorticity. How well the depicted orbit approximates a homoclinic connection can be quantified by measuring two distances: one from the end point of the computed orbit to the leading stable subspace at a point on the UPO and one to this point along the leading stable subspace. We found these distances to be of order $10^{-7}$ and $10^{-6}$ in the energy measure, respectively. To test the robustness of this result, we have recomputed the homoclinic with a smaller integration time step and a varying number of shooting intervals.

The flow structure of bursting Figure 2 shows the time-evolution of flow structures along the homoclinic orbit at the six phases indicated in Fig. 1. In the early stage of the evolution (phase a) the spanwise standing-wave motion of the streak is enhanced, so that the streamwise dependence, i.e. the three-dimensionality, of the streak gradually becomes significant. At the same time the streak grows in the wall-normal direction. Such behaviour is a direct consequence of the linear instability of
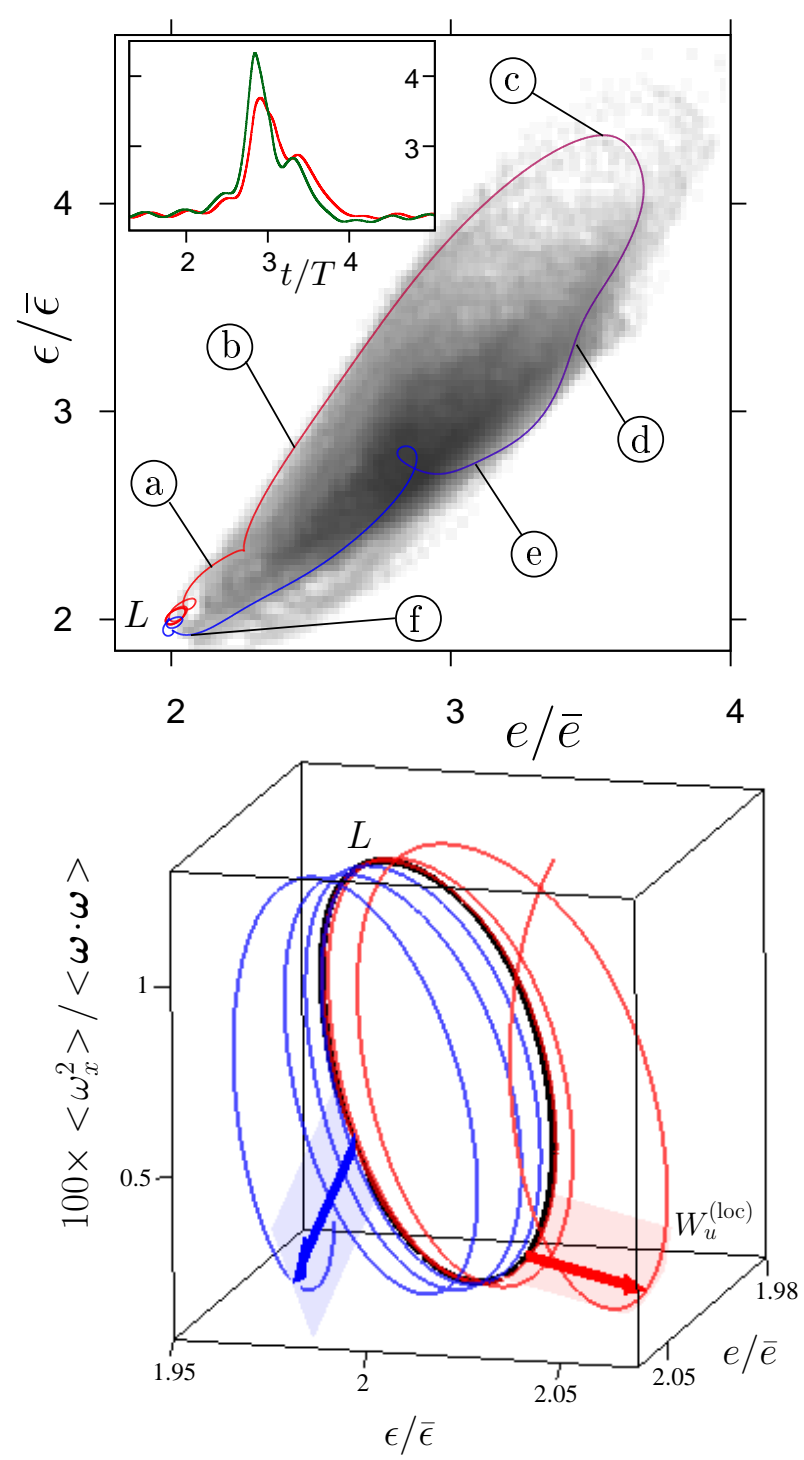

FIG. 1. Top: projection of the homoclinic orbit onto energy input and dissipation rate, normalized by their value in laminar flow, $\bar{e}$ and $\bar{\epsilon}$. The piece of orbit leaving the edge state, $L$, is shown in red and the one approaching it in blue. In the background, the PDF of transient turbulence is shown in gray scale. The labels a-f correspond to the snap shots in Fig 2 The inset shows the input (red) and dissipation (green) rate of energy as a function of time, normalized with $T$, along part of the homoclinic orbit. Bottom: close-up of the UPO, $L$, with the homoclinic. The red and the blue arrow denote the projected local unstable manifold and the projected leading stable subspace, respectively. On the axes are the normalized energy input and dissipation rate and the mean square streamwise vorticity normalized by the mean square total vorticity, where the mean is taken over the flow unit.

the UPO. The eigenstructure for the instability is characterized by disturbances of the streamwise velocity and vorticity which are highly localized on the crest and the valley of the streak.

As time progresses, the simultaneous spanwise oscillation and wall-normal growth of the streak exceed a critical 
(a)
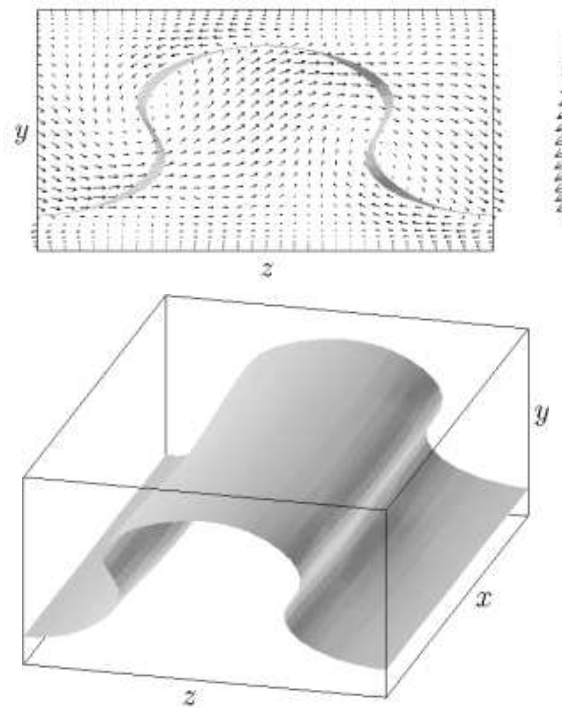

(d)
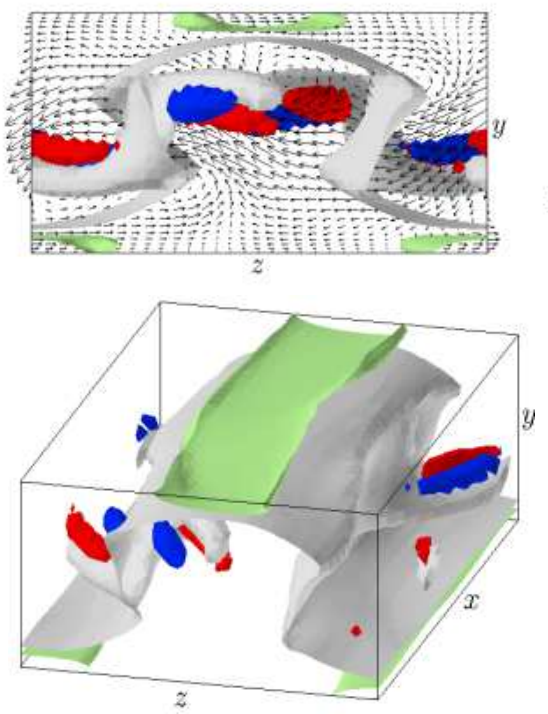

(b)
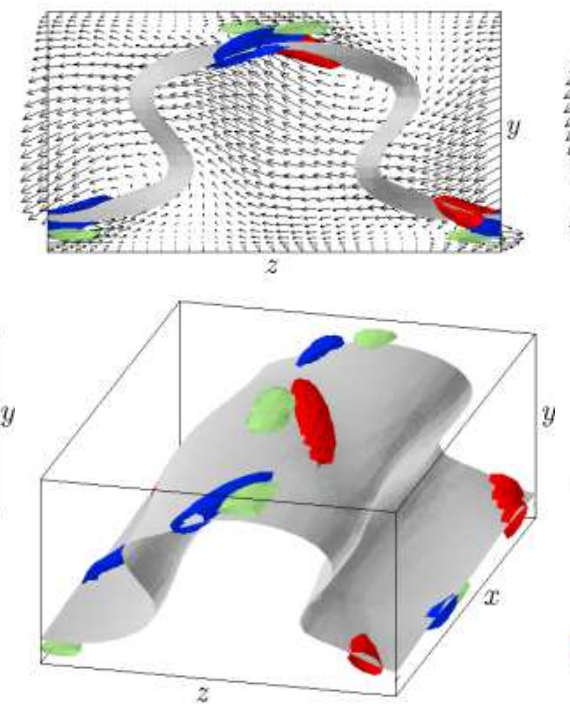

(e)
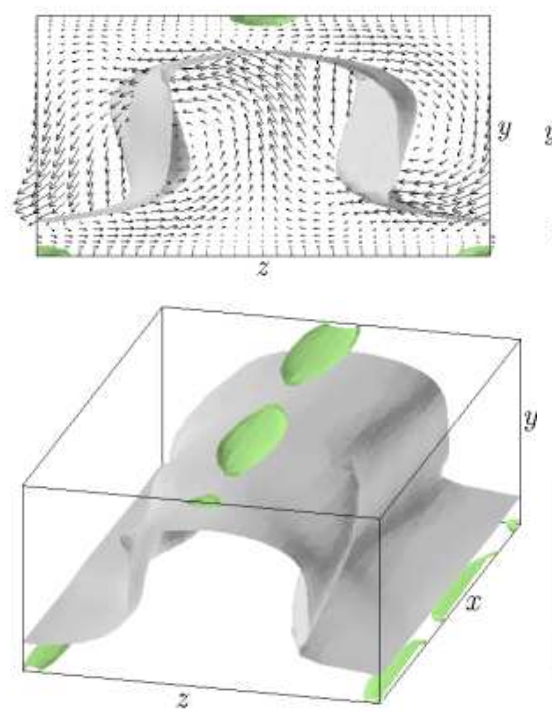

(c)
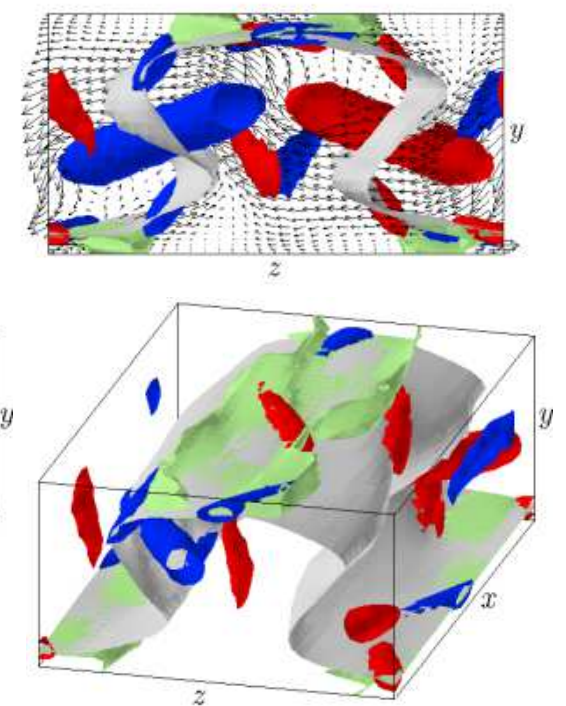

(f)
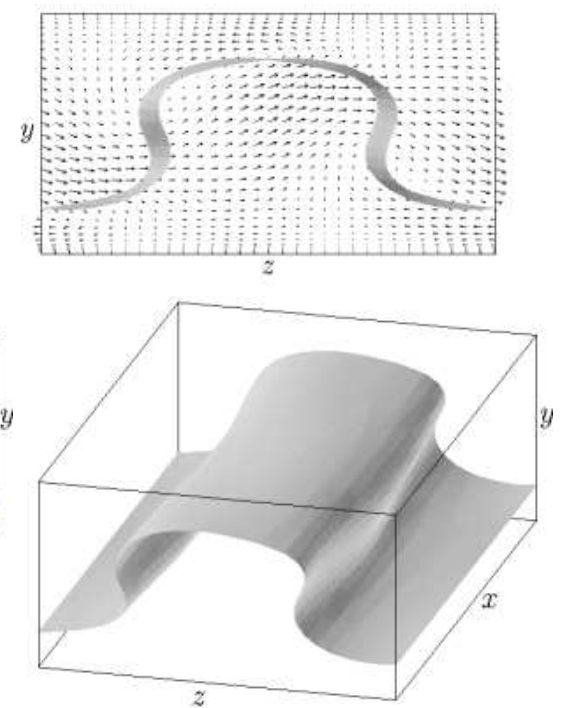

FIG. 2. Visualizations of flow structures in one periodic box $L \times W \times H$ in six phases on the homoclinic orbit, labeled as in Fig. 1. In each phase the structures are viewed in two distinct directions. Gray corrugated isosurfaces of the null streamwise $(x)$ velocity represent streamwise streaks. Red and blue objects are isosurfaces at $0.2(U / H)^{2}$ for the second invariant of a velocity gradient tensor, and denote the vortex tubes of the positive (clockwise) and negative (counter-clockwise) streamwise-vorticity component. Green isosurfaces show the local energy dissipation rate at 20 times the value $\bar{\epsilon}$ in laminar flow. Cross-stream $(y, z)$ velocity is shown in the mid plane $x=L / 2$. By the flow symmetry it is related to that at $x=0$ by a reflection in the spanwise direction.

level for bursting. There appear thin layers of extremely high vorticity between the wall and the crest and the valley of the streak, in which intense quasi-streamwise vortex tubes are generated to induce high shear and thereby high energy dissipation (phase b). The quasi-streamwise vortices of opposite signs of the streamwise vorticity align following the oscillation of the streak in the spanwise direction. Then the streak is rapidly deformed, while the second pair of quasi-streamwise vortices appears on the crest and valley, and align with the first pair to form an array which moves fast on the crest or valley in the spanwise direction (phase c). During this streak deformation and vortex generation intense energy dissipation is observed in the thin layers between the wall and the crest or the valley of the streak. Streamwise vortices similar to those found in the regeneration cycle $[\underline{6}]$ and the cor- 


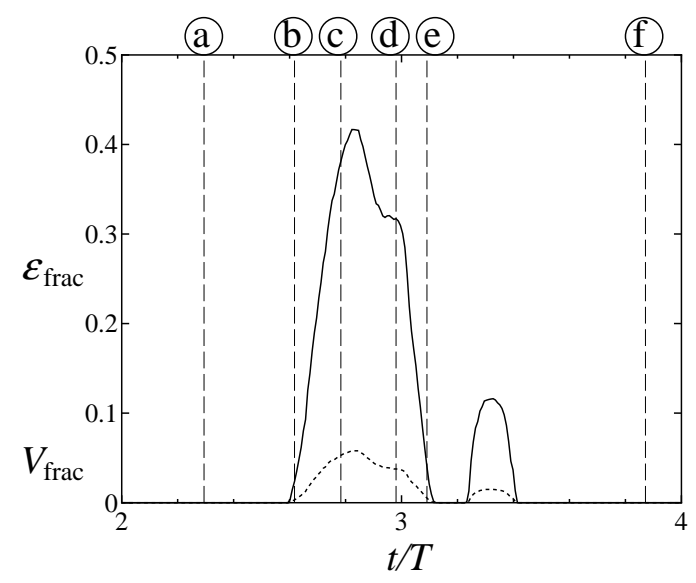

FIG. 3. Temporal variation of energy-dissipation (solid curve) and volume (dotted curve) fractions in the region bounded by the green surfaces in Fig. 2 where the local dissipation rate is greater than 20 times the value in laminar flow. Time $t$ is normalized with the period of the gentle UPO, and the six phases on the orbit are shown by dashed vertical lines.

responding strong UPO [9] also appear on the flanks of the streak. However, the streamwise vortices on the crest and valley of the streak are not observed in the near-wall regeneration cycle, and therefore we can say that these vortices are typical flow structure of bursting with significant energy dissipation. Actually, an inspection of the turbulent state of plane Couette flow has shown that in bursting events of intense dissipation there appear similar vortex arrays on the crest and valley of the highly grown streak, which are associated with strong local dissipation. As shown in Fig. 3, the intense local dissipation (represented by green isosurfaces) observed around the crest and valley of the streak in Fig. 2 (phases c and d) contributes to the rapid increase of total energy dissipation (see Fig. 1). The identified high-dissipation regions occupy less than $10 \%$ of the spatial domain but account for over $40 \%$ of the energy dissipation, implying that strong energy dissipation in the bursting event can be attributed to flow structures localized in the region around the crest and the valley of the streak shown by the green surfaces in Fig. 2. As time goes on, the highly deformed streaks are broken down (phase d), and then the streak and vortices decay rapidly (phase e) as the flow finally returns to a quiescent state close to the gentle UPO (phase f).

Transient approaches of a turbulence state to relatively quiescent states of low energy input and dissipation are followed by bursting events with intense energy dissipation, as reported by Kawahara \& Kida [9], who suggested the interpretation of bursting in terms of a twoway heteroclinic connection between the gentle UPO and a UPO embedded in turbulent flow. The present more rigorous analysis has presented another interpretation, namely that the whole process of the bursting, including a transient approach to a quiescent state and a subsequent highly dissipative event, is the manifestation of a homoclinic orbit arising from the gentle UPO alone.

Conclusion The existence of an orbit homoclinic to this edge state implies that the geometry of the laminarturbulent boundary is rather complex and we can expect to find a manageable approximation to it only locally. At the same time, it implies the existence of infinitely many UPOs which correspond to flows with arbitrarily many, arbitrarily long, near laminarization events. It is natural then to think of turbulent shear flow as governed by a large chaotic attractor which comprises both periodic orbits in the turbulent regime, which reproduce the regeneration cycle [9], and periodic orbits which reproduce near-laminarization and bursting events. Physically, these bursting events are very different from the regeneration cycle. The streamwise vortices appear on the crest and valley of the streaks rather than on their flanks, and the larger part of the energy dissipation takes place in the cycle around the vortices in the near-wall, high strain region.

The homoclinic solution presented here adds a new element to the elucidation of turbulence by means of dynamical systems theory, namely that of temporal localization. Since it has been computed in a minimal flow unit, in cannot capture spatial intermittency. Recently, several equilibrium and travelling wave solutions exhibiting spatial localization have been found [11]. A clear goal for the near future is to find periodic or connecting orbits which combine the two forms of localization.

* lennaert.vanveen@uoit.ca; Supported by NSERC Grant nr. 355849-2008

† kawahara@me.es.osaka-u.ac.jp Supported by JSPS Grant-in-Aid for Scientific Research

[1] T. Itano and S. Toh, J. Phys. Soc. Jpn. 70, 703 (2001); J. Fluid. Mech. 482, 67 (2003).

[2] With pipe flow as celebrated example: Hof, B. et al., Nature 305, 1594 (2004).

[3] See, e.g., B. Eckhardt, H. Faisst, A. Schmiegel, and T. M. Schneider, Philos. T. Roy. Soc. A 366, 1297 (2008).

[4] G. Kawahara, Phys. Fluids 17, 041702 (2005).

[5] S. Smale, in Differential and combinatorial topology (Princeton University Press, 1965) pp. 63-80.

[6] J. M. Hamilton, J. Kim, and F. Waleffe, J. Fluid Mech. 287, 317 (1995).

[7] M. Nagata, J. Fluid Mech. 217, 519 (1990).

[8] J. Jiménez, G. Kawahara, M. P. Simens, M. Nagata, and M. Shiba, Phys. Fluids 17, 015105 (2005).

[9] G. Kawahara and S. Kida, J. Fluid Mech. 449, 291 (2001).

[10] L. van Veen, G. Kawahara, and A. Matsumura, SIAM J. Sci. Comput. 33, 25 (2011).

[11] T. M. Schneider, J. F. Gibson, and J. Burke, Phys. Rev. Lett. 104, 104501 (2010); U. Ehrenstein, M. Nagata, and F. Rincon, Phys. Fluids 20, 064103 (2008). 